\title{
Exposure of the Plasmodium falciparum clonally variant STEVOR proteins on the merozoite surface
}

\author{
Ayman Khattab*, Seppo Meri
}

\begin{abstract}
Background: Plasmodium falciparum merozoites are free invasive forms that invade host erythrocytes in iterative cycles in the presence of different arms of the immune system. Variant antigens are known to play a role in immune evasion and several gene families coding for variant antigens have been identified in $P$. falciparum. However, none of them have been reported to be expressed on the surface of merozoites.
\end{abstract}

Methods: Flow cytometry, immunofluorescence microscopy, and immunoblotting assays were performed to assess surface exposure, membrane association and stage specific expression of the STEVOR family of variants proteins, respectively.

Results: Using a polyclonal antibody (anti-PFL2610w) with a broad specificity towards different STEVOR variants, the STEVOR proteins were identified on the surface of non-permeabilized/non-fixed merozoites in flow cytometry assays. Anti-PFL2610w antibody showed that several STEVORs were expressed in the trophozoite stage of the parasite but only one variant was integrated into the merozoite membrane. Moreover, this antibody failed to identify STEVORs on the surface of the parent schizont infected erythrocytes (IE) although they were readily identified when schizont IE were permeabilized.

Conclusions: These data suggest for a role for STEVOR in immune evasion by P. falciparum merozoites to allow successful invasion of erythrocytes. Additionally, the expression of STEVORs in the schizont stage may only represent a step in the biogenesis process of the merozoite surface coat.

\section{Background}

Of the four species causing human malaria, Plasmodium falciparum is the most dangerous. It causes the highest rates of complications and mortality in the tropical and subtropical regions worldwide. It was estimated for the year 2002 that about 500 million people suffered from P. falciparum-malaria and about one million died [1]. The remarkable ability of the $P$. falciparum parasite to achieve this in comparison to other Plasmodium species is due to its capability to infect all ages of human erythrocytes leading to a higher parasite load. Additionally, the P. falciparum trophozoite and schizont IE stages have a unique competence to cytoadhere to a variety of human endothelia to escape from the circulation and avoid clearance by the spleen until merozoites become released and invade new erythrocytes. In malaria endemic areas, immunity develops against mild or

\footnotetext{
* Correspondence: ayman.khattab@helsinki.fi

Malaria Research Laboratory, Department of Bacteriology and Immunology, Haartman Institute, University of Helsinki, Helsinki, Finland
}

uncomplicated P. falciparum malaria over a long period of time during which individuals are repeatedly infected with the parasite. On the other hand, immunity against severe forms of malaria such as severe anaemia and cerebral malaria take shorter periods to develop. A broad immunity to most forms of malaria is reached at adulthood and declines when an individual leaves the endemic area [2-4]. Despite acquiring immunity to malaria, the parasite persists and causes an asymptomatic infection in adults living in areas where malaria is endemic. Thus infected adults serve as a reservoir for the parasite in hyperendemic areas where malaria is seasonal and mosquitoes disappear during the dry seasons.

Successful merozoite invasion of new erythrocytes in the iterative growth cycle of the P. falciparum parasites is a prerequisite for parasite persistence in adults in endemic areas. This appears to occur despite acquiring immunity that limits both severe and mild forms of malaria. It also indicates that the parasite must have effective tools to complete the invasion steps (attachment, reorientation,

\section{Biomed Central}


engulfment and entry) without being harmed by the immune system. Proteins that possess highly variable sequences and encoded by multi-copy gene families are lead candidates for such tools. The var, rif and stevor are known $P$. falciparum multi-copy gene families that undergo antigenic variation [5], they could thus be involved in merozoite escape from the immune system attack.

STEVORs encoded by the stevor gene family are the most widely expressed variant antigens in the different stages of the $P$. falciparum parasite. They were found in the late trophozoite and schizont IE stages in flattened vesicular structures known as Maurer's clefts (MC) localized in the erythrocyte cytoplasm [6]. Further studies localized STEVORs to the IE membrane by electron microscopy $[7,8]$ or to the IE surface by flow cytometry [9]. STEVORs were also detected in the gametocyte and sporozoite stages of the parasite [10]. The stevor family is represented by 39 members in the 3D7 genome [11]. stevor gene transcription peaks at the mid-trophozoite stage [6], $28 \mathrm{~h}$ post invasion (p.i.) and only a subset of stevor genes are transcribed at any given time in a population of parasites [12]. Evidence for clonal variation in stevor expression was reported at the transcription level [12] and at the protein level [8,9]. STEVORs share protein architecture with proteins encoded by the rif and the Pfmc-2TM families and altogether constitute what is described as the TM superfamily [13]. This architecture consists of a signal sequence, a hypervariable (HV) region surrounded by two predicted transmembrane domains and a cytoplasmic tail. The HV region is of varying length and up to 170 and 60 amino acid residues in RIFINs and STEVORs, respectively and only three residues, on average, in the Pfmc-2TM family [13]. Members of the TM superfamily have no known function so far. In recent work evidence was presented by our group for the expression of STEVORs in the merozoite stage of the P. falciparum parasite. Immunofluorescence and immunoelectron microscopy localized STEVORs to the surface coat of the merozoites and to the rhoptries [8]. Localization of STEVORs to the rhoptries was also shown in a different study [14]. These data suggest that the parasite might utilize STEVORs expressed in the merozoite stage to vary antigenically as a means to establish a long-lasting persistent infection together with similar or different strategies in the other stages of the parasites. Since the accessibility of the variant antigen to the surface of the cell is an important feature in mediating immune evasion, a follow-up study was conducted to verify the exposure of STEVORs on the merozoite surface.

Here, a polyclonal anti-STEVOR antibody (antiPFL2610w) identified STEVOR(s) on the surface of merozoites in flow cytometry assays. Similar variants were identified by the same antibody also in the parent schizont IE, but not on the surface of the intact schizont IE. Western blot analyses of protein extracts from early ring, mid-trophozoite and merozoite stages of the parasite provided evidence on the over-expression of a single STEVOR variant in merozoites. These data strengthen the suggested role of STEVORs in mediating immune evasion by the merozoites to acquire a long lasting capability of invading host erythrocytes.

\section{Methods}

\section{Parasite strain and culture}

The $P$. falciparum strain used in this study was the 3D7 parasite line. It was cultured in $\mathrm{O}^{+}$human erythrocytes at 5\% haematocrit in RPMI-1640 medium supplemented with 0.5\% Albumax II (Gibco, Carlsbad, CA, USA), $200 \mu \mathrm{M}$ hypoxanthine (Sigma, St. Louis, MO, USA) and $20 \mu \mathrm{g} / \mathrm{mL}$ gentamycin (Gibco).

\section{Anti-STEVOR antibody}

Mouse polyclonal Anti-STEVOR antibody was generated [15] against a recombinant STEVOR that included both the hypervariable and the conserved region of the PFL2610w stevor gene (Figure 1). Amino acid boundaries of the recombinant protein are described elsewhere [15].

\section{Schizont IE enrichment and isolation of free $P$. falciparum merozoites}

Erythrocytes infected with $P$. falciparum ring stages were synchronized twice, $4 \mathrm{~h}$ apart, by sorbitol treatment (Sigma, St. Louis, MO, USA), grown to high parasitaemia $(\approx 10 \%)$ of mostly schizonts and enriched to a parasitaemia of about $90-95 \%$ by magnet-activated cell sorting (MACS). To prepare naturally released merozoites, 44-48 hour (p.i.) schizont IE were purified by MACS and placed back into culture at $1 \%$ haematocrit in RPMI-1640 complete medium (P. falciparum culture medium). Every $90 \mathrm{~min}$ the culture was examined by microscopy for schizont IE rupture and merozoite release. When the majority of schizont IE had ruptured, free haemozoin, intact schizont IE and uninfected erythrocytes were removed by centrifugation for $4 \mathrm{~min}$ at $600 \times \mathrm{g}$ at room temperature followed by a single step of purification by passage through the MACS column. Finally, merozoites were pelleted from the flow through

\begin{tabular}{|l|l|l|l|l|}
\hline SP & TM1 & HV & TM2 & CT \\
\hline Figure 1 Domain architecture of the STEVOR protein family. \\
The recombinantly expressed STEVOR used to produce the anti- \\
PFL2610w antibody is delineated by the arrows. Abbreviations: \\
signal peptide (SP), conserved region (CR), hypervariable region (HR), \\
transmembrane domain (TM) and cytoplasmic tail (CT). \\
\hline
\end{tabular}


by centrifugation for $15 \mathrm{~min}$ at $2880 \times \mathrm{g}$ at $4^{\circ} \mathrm{C}$, and washed once in PBS. Merozoite integrity was checked by Giemsa staining. Merozoites used in immunoblotting were further purified by double filtering through $3 \mu \mathrm{m}$ and $1.2 \mu \mathrm{m}$ Versapor membranes and pelleted by centrifugation for $15 \mathrm{~min}$ at $2880 \times \mathrm{g}$ at $4^{\circ} \mathrm{C}$.

\section{Immunofluorescence and colocalization assays}

For indirect immunofluorescence microscopy, parasites were cultured and synchronized as described above and allowed to grow for one cycle before samples were processed. IE representing the schizont stage were smeared on glass slides, air-dried and fixed for $5 \mathrm{~min}$ with icecold methanol. Smears were blocked with PBS/1\% BSA for $30 \mathrm{~min}$ at room temperature. Slides were washed once in PBS/0.05\% Tween 20 for $5 \mathrm{~min}$ and incubated at room temperature for $2 \mathrm{~h}$ with mouse anti-PFL2610w antibody (1:500) or a mix of mouse anti-PFL2610w antibody (1:500) and rabbit anti-MSP1 antibody (1:200) in $\mathrm{PBS} / 1 \% \mathrm{BSA}$. Slides were then washed $3 \times 5 \mathrm{~min}$ in PBS $/ 0.05 \%$ Tween 20. Binding of the primary antibodies was visualized by Alexa 488-conjugated goat anti-mouse IgG (1:1000; Molecular Probes) when anti-PFL2610w antibody was solely used, whereas a mixture of Alexa 488-conjugated goat anti-mouse (1:1000; Molecular Probes) and Alexa 546-conjugated goat anti-rabbit (1:1000; Molecular Probes) were used when combining the analysis of both anti-PFL2610w and anti-MSP1 primary antibodies. Cell nuclei were visualized by DAPI (4',6-diamidino-2-phenylindole) $(5 \mu \mathrm{g} / \mathrm{ml}$; Roth, Germany). Slides were mounted on MOWIOL (Calbiochem, San Diego, CA, USA), viewed with the Olympus BX51 fluorescence microscope and images were captured using the Olympus DP70 camera with the help of DP controller software. Pre-immune sera were used to replace the anti-PFL2610w antibody for the negative control slides.

\section{Flow cytometry assays}

Enriched schizont IE and purified free merozoites were tested for recognition of their surface by the antiPFL2610w antibody. Schizont IE and merozoite preparations were diluted to approximately $5 \times 10^{6} \mathrm{cells} / \mathrm{ml}$ in PBS/1\% BSA. Nuclei of both stages were stained with $20 \mu \mathrm{l}$ ethidium bromide $(0.1 \mathrm{mg} / \mathrm{ml}$ in PBS) per $\mathrm{ml}$ of cell suspension. The anti-PFL2610w antibody and the pre-immune serum were diluted to 1:400 in $100 \mu \mathrm{l}$ of cell suspensions and incubated at $4^{\circ} \mathrm{C}$ for $1 \mathrm{hr}$. Schizont IE and merozoites were then washed twice in $1.5 \mathrm{ml}$ $\mathrm{PBS} / 1 \% \mathrm{BSA}$ and pelleted after each wash by centrifugation at $4^{\circ} \mathrm{C}$ for $5 \mathrm{~min}$ at $800 \times \mathrm{g}$ and $6000 \times \mathrm{g}$, respectively. Schizont IE and merozoites were resuspended in $100 \mu \mathrm{l}$ Alexa 488-conjugated goat anti-mouse diluted to 1:500 in $\mathrm{PBS} / 1 \% \mathrm{BSA}$ and incubated at $4^{\circ} \mathrm{C}$ for $1 \mathrm{hr}$ and then washed as above. Stained schizont IE and merozoites were resuspended in $200 \mu \mathrm{lBS} / 1 \% \mathrm{BSA}$ and analysed using the FACScan flow cytometer. Flow cytometry assays on permeabilized schizont IE were also performed. Treatment of packed schizont IE with 10 volume of $0.05 \%$ saponin in PBS for $10 \mathrm{~min}$ on ice renders the erythrocyte membrane freely permeable to solutes as large as soluble proteins (e.g. antibodies). Permeabilized schizont IE were washed thrice with the permeabilization buffer, pelleted after each wash by centrifugation at $3000 \times \mathrm{g}$ for $5 \mathrm{~min}$ at $4^{\circ} \mathrm{C}$ and treated with the same flow cytometry staining protocol as described above. Buffers used for primary and secondary antibody incubation and for washing included 0.05\% saponin.

\section{Western blot analysis}

Merozoite and saponin permeabilized early ring-IE (8-12 h) and mid-trophozoite-IE (24-30 h) stages representing a single growth cycle of the synchronized parasite were analysed by immunoblotting for the determination of STEVOR expression pattern. Trophozoite-IE and ringIE stages were permeabilized as described above and the final pellets were boiled in $1 \times$ SDS sample buffer for $10 \mathrm{~min}$. A fraction of each protein extract was loaded onto SDS-polyacrylamide gels and separated proteins were transferred to Trans-Blot transfer medium membrane (Bio-Rad, Hercules, CA, USA). The presence of erythrocyte membranes in each protein extract preparation was monitored with anti-glycophorin A antibody (abcam, USA). The membranes were probed with either anti-PFL2610w antibody or the pre-immune serum. Reactivity was visualized by goat anti-mouse IgG coupled to horseradish peroxidase (1:30 000; Pierce, Rockford, IL, USA). Blots were developed by the enhanced chemiluminescent (ECL Western blot analysis system)-based detection according to the manufacturer's instructions (GE Healthcare, Life Sciences, Bucks, UK) followed by exposure to Super RX films (Fujifilm). Blots were imaged and bands were quantified by densitometric analysis of band intensity using Kodak $1 \mathrm{D}$ v. 3.5.3 software (Kodak Scientific Imaging Systems).

\section{Results}

Infected erythrocytes permeabilization is required for STEVOR recognition by the anti-PFL2610w antibody in flow cytometry assays

The predicted secondary structure characteristics of STEVORs suggest that STEVORs' hypervariable region could acquire cell surface location and be exposed to the immune system at some stages during infection. Thus, STEVORs are speculated to be involved in antigenic variation and host parasite interaction, biological functions that are well described for the IE surface 
protein, PfEMP1. This is known to mediate cytoadhesion and immune evasion in $P$. falciparum infection. Earlier reports showed expression of STEVORs in the IE as well as other stages of the parasite. More recent studies have shown the expression of STEVORs at the apical end of the merozoites in immunofluorescence microscopy and on the surface of IE using flow cytometry assays and STEVOR variant-specific antibodies $[9,14]$. In our parallel effort to link the predicted protein organization of STEVORs with the biology and pathogenesis of the parasite, STEVORs were shown to be associated with the surface of merozoites [8]. STEVORs were also detected as released proteins from the merozoites during erythrocyte invasion [8]. These findings prompted us to ask whether the variant specific antibody that was used to localize STEVORs at the merozoite membrane can identify STEVORs on the surface of the parent schizont IE or a biologically relevant stage-specific differential expression is taking place. Thus, enriched non-permeabilized/non-fixed 3D7 schizont IE were analysed in flow cytometry assays using the antibody that we have produced against a specific STEVOR variant (PFL2610w). This antibody is capable of recognizing STEVORs associated with the merozoite membrane. Minimal binding of anti-PFL2610w antibody to the surface of schizont IE was observed as indicated by the slight shift in the relative fluorescence intensity compared to the basal level binding of the pre-immune serum antibodies (Figure 2A, lower panel). This was confirmed by immunofluorescence microscopy on the same preparation (Figure 2A, upper panel). Additionally, the immunofluorescence microscopy showed some green fluorescent staining that did not overlay well with the nuclear staining. This probably represented a population of ruptured cells. This matched the dot blot quadrant statistics showing an increase in the ethidium bromide/Alexa-488 double stained population from $2.17 \%$ with pre-immune serum to $3.86 \%$ with the antiPFL2610w antibody (dot plot figure not shown). This observation prompted us to ask whether the antiPFL2610w antibody could recognize intact cells if they are permeabilized, a condition close to cell rupture. Consequently, schizont IE were permeabilized by $0.05 \%$ saponin and the staining protocol was repeated as before but with the exception that $0.05 \%$ saponin was present in all solutions. Staining protocol was also performed on non-permeabilized schizont IE to control for possible generation of specific variations in the expressed STEVORs. Interestingly, flow cytometry analysis of this preparation showed a striking shift in the relative fluorescence intensity compared to the pre-immune serum (Figure 2B, lower panel). The same preparation was also analysed by immunofluorescence microscopy and schizont IE staining with anti-PFL2610w was confirmed (Figure 2B, upper panel). On the other hand, the anti-PFL2610w antibody still did not bind to the non-permeabilized schizont IE. In conclusion, these findings indicated that the anti-PFL2610w can only recognize STEVOR variants that are associated with internal schizont IE structures but not those presumably expressed on the surface.

\section{STEVOR is expressed on the surface of merozoites}

Next we wanted to study whether anti-PFL2610w antibody can recognize STEVORs in merozoites released from schizont IE that were used for the flow cytometry assay and did not show any surface labelling. A fraction of this schizont IE preparation was allowed to naturally rupture and release merozoites. Subsequently, binding of anti-PFL2610w antibody to merozoite membranes was assessed by immunofluorescence microscopy using methanol fixed merozoite slides. Remarkably, antiPFL2610w antibody that failed to recognize the surface of non-permeabilized schizont IE in flow assays readily recognized the membranes of fixed merozoites in immunofluorescence microscopy (Figure 3). Moreover, an antibody to the well described merozoite surface protein (MSP-1) showed that both proteins occupy the same location (Figure 3). Additionally, recognition of STEVOR variant(s) in the merozoite membrane by this antibody clearly showed the association of STEVORs with the merozoite surface membrane. However, it remained unclear whether STEVORs were exposed on the surface or not, a location suspected for an antigen with such domain organization like STEVOR. To further clarify these points freshly isolated nonpermeabilized/non-fixed merozoites (purity of the preparation is shown in Figure 4A) were probed with anti-PFL2610w antibody and the bound antibodies were visualized by anti-mouse Alexa- 488 conjugated antibody. Interestingly, anti-PFL2610w antibody bound to the surface of merozoites represented as the Ethidium bromide/Alexa 488 double stained population (Figure $4 \mathrm{C}$, lower panel). Antibodies in the pre-immune serum did not show any significant binding to the merozoite surface (Figure 4B, lower panel) supporting the specificity of the anti-PFL2610w binding. Altogether, these data showed that anti-PFL2610w could not recognize STEVOR on the surface of the schizont IE, but rather in internal structures. Moreover, STEVORs were readily indentified on the merozoites surface upon their release from the schizont IE.

\section{Over-expression of a single STEVOR in merozoites}

Anti-PFL2610w was generated against a recombinant protein that included both the variable variant specific sequence and the semi-conserved STEVOR family specific sequence (Figure 1). This antibody was successfully 
A
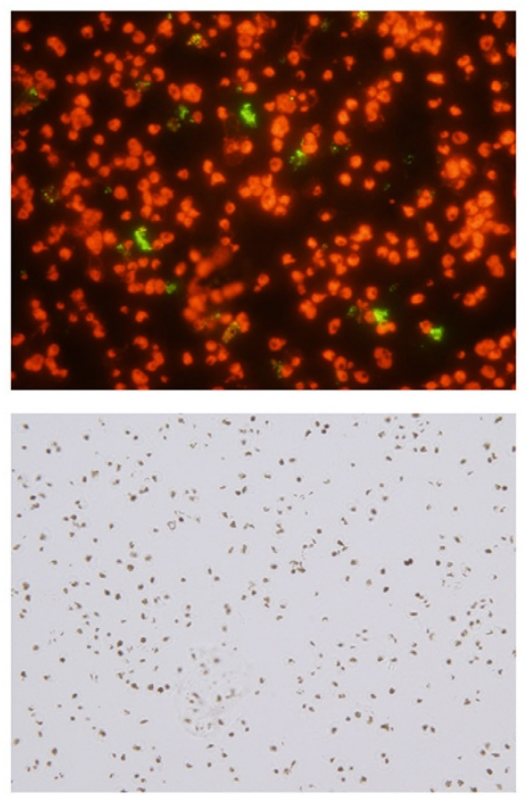

กิ

Non-permeabilized schizonts

แั๊

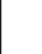

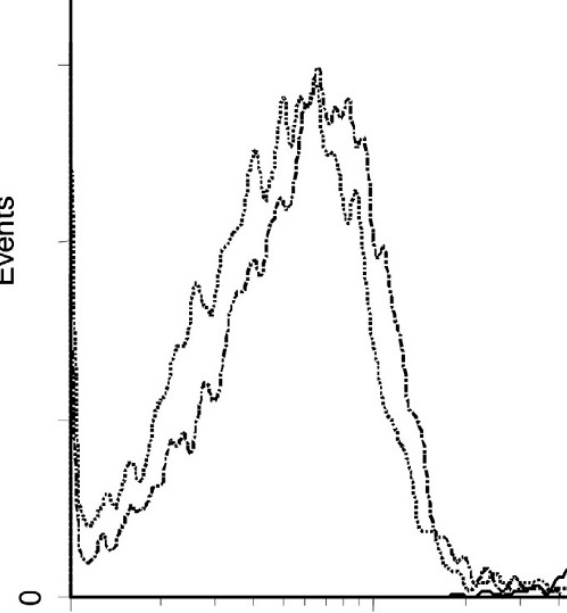

$10^{0}$

$10^{1}$
B
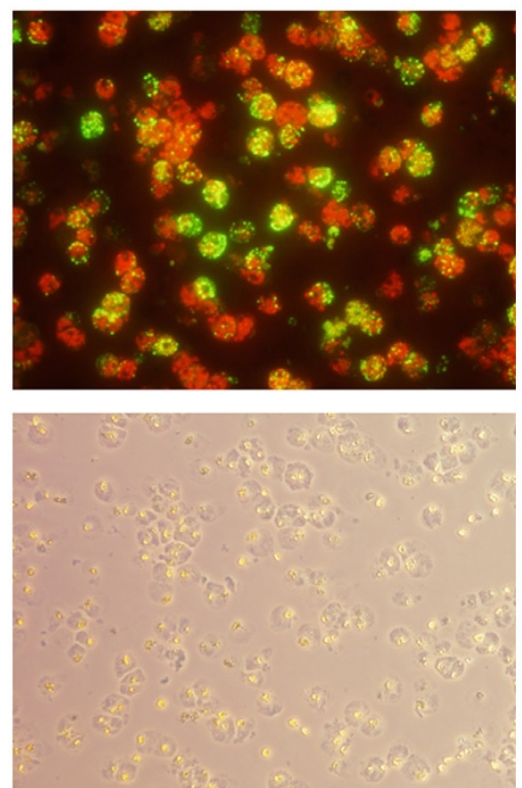

Permeabilized schizonts

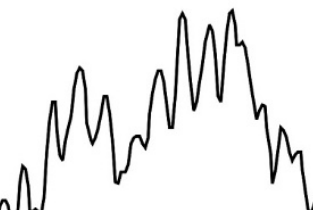<smiles>C1=[V]C[Te]1</smiles>

Figure 2 Recognition of STEVORs in non-permeabilized and saponin-permeabilized schizont infected erythrocytes (IE). (A, lower panel) Flow cytometry histogram of non-permeabilized schizont IE recognized by the pre-immune serum (dot line) and by the anti-PFL2610w antibody (dot-dash line). (A, upper panel) Fluorescence microscopy image of the schizont IE that were recognized by anti-PFL2610w antibody in flow cytometry. (B, lower panel) Flow cytometry histogram of permeabilized schizont IE recognized by the anti-PFL2610w antibody (solid line). (B, upper panel) fluorescence microscopy image of the permeabilized schizont IE recognized by anti-PFL2610w antibody in flow cytometry.

used to study clonal variation of STEVOR expression in NF54-derived lines in relation to their binding capacity to chondroitin sulphate A and CD36 receptors and subsequent loss of these phenotypes [8]. Therein, several STEVOR variants were identified at the late trophozoite stage by the anti-PFL2610w antibody in Western blot analyses. Moreover, the antibody identified STEVOR expression in the ring, trophozoite, schizont and merozoite stage parasites in immunofluorescence microscopy. The former data and the new finding on STEVOR expression on the surface of merozoites prompted us to ask whether a differential expression of STEVOR 

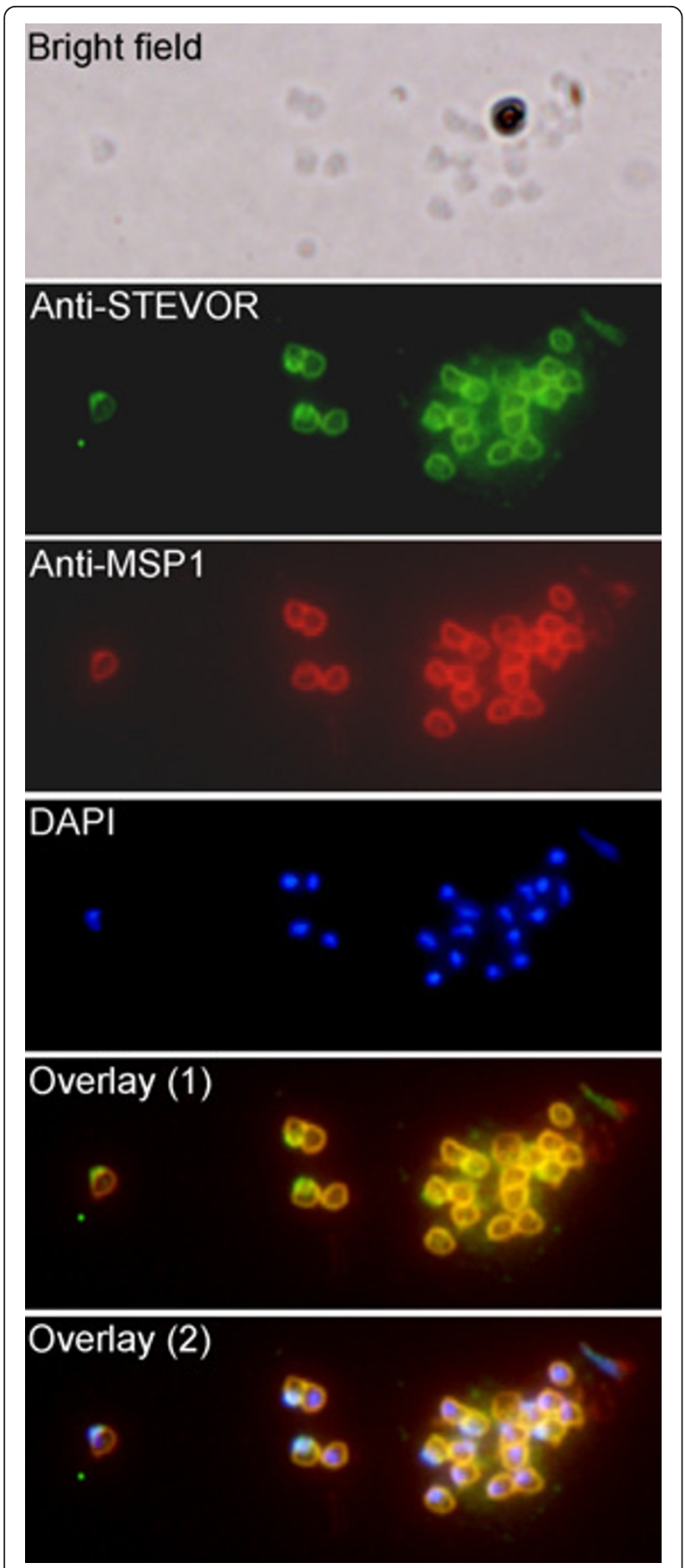

Figure 3 Colocalization of STEVORs with MSP-1 at the free merozoite membranes. Fluorescence staining using anti-PFL2610 W and anti-MSP-1 antibodies was analysed in free merozoites. (A) Bright field, (B) Alexa 488 stained STEVORs, (C) Alexa 594 stained MSP-1, (D) DAPI stained parasite nuclei, (E) the overlay of STEVORs and MSP-1 (overlay 1) and (F) the overlay of STEVORs, MSP-1 and nuclei (overlay 2) images are shown. variants take place in these parasite stages. Therefore, SDS-soluble proteins of the early ring (8-12 h), mid-trophozoite (24-30) and merozoite stages corresponding to a single generation were analysed in a Western blot analysis using the anti-PFL2610w anti-STEVOR antibody. A total of five STEVOR variants with variable band intensities were identified by the antibody (Figure 5A and Table 1) in the stages examined. Only two variants (31.7 and $29.8 \mathrm{kDa}$ ) with relative band intensities of 0.56 and 0.44 respectively were identified by the antiPFL2610w antibody in the early ring stage parasite (Figure $5 \mathrm{~A}$ and Table 1). The transition from ring to trophozoite stage was accompanied by the expression of three more variants $(34,28$ and $25 \mathrm{kDa})$ in addition to the two ring stage variants $(31.7$ and $29.8 \mathrm{kDa})$. These two variants retained relatively higher expression levels compared to the other variants according to the relative band intensities estimation (Figure 5A and Table 1). Interestingly, the development of mid-trophozoites into schizonts for merozoite release resulted in over-expression of a single variant $(31.7 \mathrm{kDa})$ in the free merozoites (Figure 5A and Table 1). This merozoite specific variant $(31.7 \mathrm{kDa})$ was one of the five variants that seemed to have nearly equal expression levels in the mid-trophozoite stage. Possible scenarios for these differences in the levels of expression between different stages include stage specific upregulation of gene expression or compartment specific localization of the expressed proteins. The latter scenario implies selective integration of specific STEVOR variants into merozoite membranes during their biogenesis. To guarantee that the merozoite preparation was devoid of IE membranes, merozoites were purified by double filtering through $3 \mu \mathrm{m}$ and $1.2 \mu \mathrm{m}$ Versapor membranes. This ensured that IE membranes from ruptured schizont IE were not carried over to the merozoite preparation. This was verified by detecting the glycophorin A (GPA) content in the Western blot (Figure $5 \mathrm{~B}$ ) using sample volumes identical to those used for detecting STEVOR variants. Anti-GPA detected only a minor quantity of GPA dimmer in the merozoite preparation with a band intensity value of $31.5 \mathrm{com}-$ pared to 209, 201 and 166 for uninfected E, ring IE and trophozoite IE respectively (Figure $5 \mathrm{~B}$ ). Monomeric GPA and GPA/Glycophorin B (GPB) complex were undetectable in the merozoite preparation (Figure 5B). In conclusion, a single STEVOR variant appeared to be over represented in the merozoite stage from in vitro culture of the 3D7 P. falciparum parasite.

\section{Discussion}

Parasitic protozoa are unicellular eukaryotic pathogens that reside in cells and/or in the extracellular fluids of 

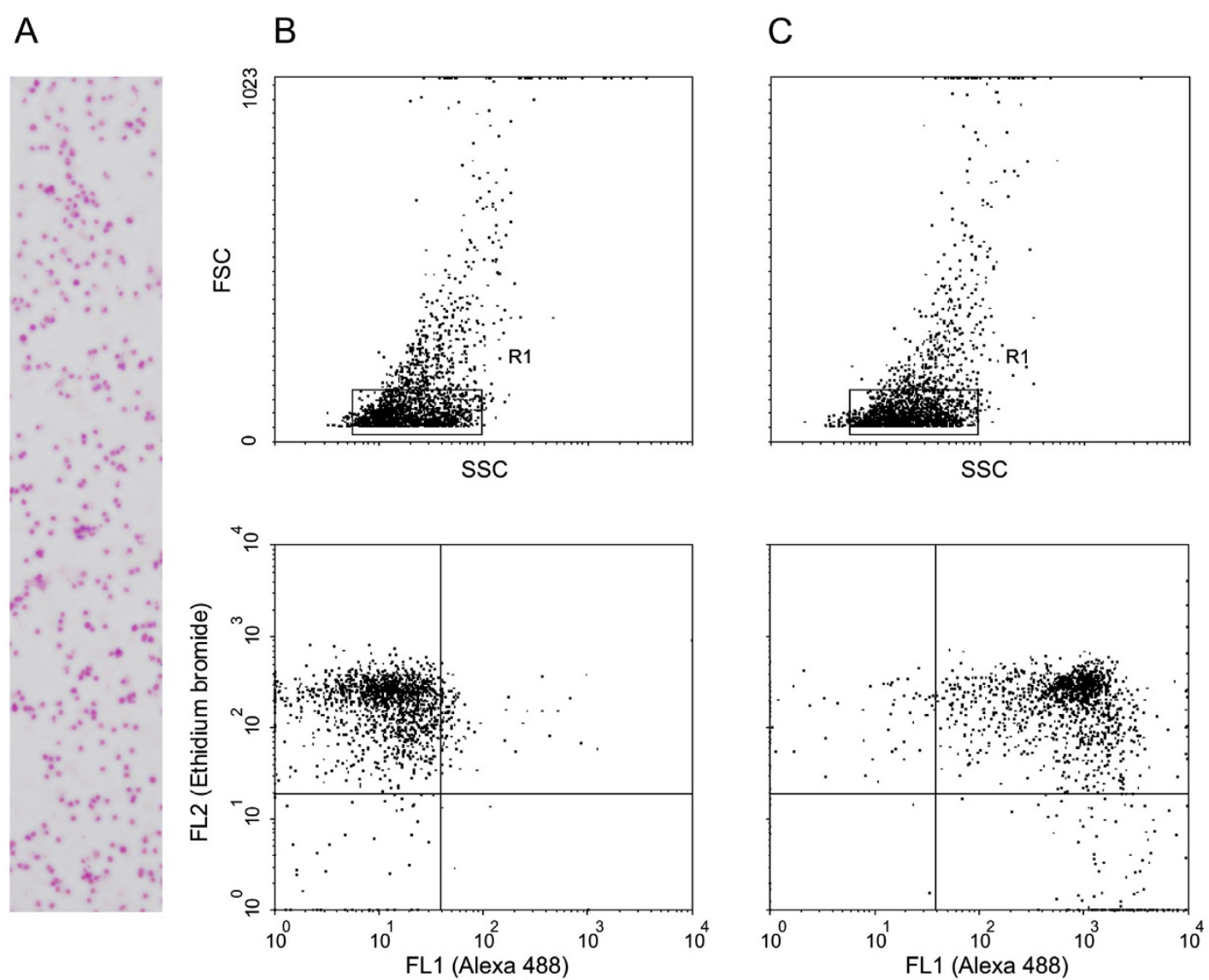

Figure 4 Detection of STEVORs on the surface of merozoites by flow cytometry. (A) Merozoite preparation stained with Giemsa. (B and C, upper panels) Dot-plots of forward scatter (FSC) to side scatter (SSC), merozoite populations are surrounded by the R1 gate. (B and C, lower panel) Dot-plots of Ethidium bromide- to Alexa 488-derived fluorescence of the gated populations. (C, lower panel) double-positive merozoite population demonstrating surface exposure of STEVORs at the upper right quadrant.

their hosts. Their success as parasite pathogens depends on a series of complicated and highly evolved host adaptations that enable them to avoid destruction by the immune system. Adoption of an intracellular life cycle is perhaps the simplest solution for evading antibody responses. Another major strategy that protects both intracellular and extracellular protozoan pathogens from immune recognition is antigenic variation of proteins expressed on the surface of the pathogen. Plasmodium parasites are among those protozoa that utilize both strategies. On one hand, they acquired both an intracellular and extracellular life style and, on the other hand, they display variant antigens on the surface of some parasite stages. Antigenic diversity on the surface of P. falciparum IE is well represented by PfEMP1 that undergoes antigenic variation while maintaining ability to cytoadhere. There is compelling data on the exposure of this molecule on the surface of the infected erythrocyte $[16,17]$.
Other antigens also classified as P. falciparum variant antigens are encoded by the multi-copy rif and stevor gene families. Analogous to what has been described for PfEMP1, original studies have reported the expression of RIFINs, encoded by the rif gene family, in internal structures and on the surface of the late blood stages of the parasite by the surface iodination technique [18]. While a recent report described the expression of RIFINs in a cap-like structure at the apical end of the merozoite [19], additional evidence to support the previously described surface expression of RIFINs is still lacking. STEVORs comprising another putative variant antigen family are expressed in the trophozoite, schizont, merozoite, gametocyte and sporozoite stages. They are thought to be also displayed on the cell surface; a location that would justify their amplification into a hypervariable multigenic family. A recent study showed the expression of STEVOR on the surface of late schizont using antibodies against specific 


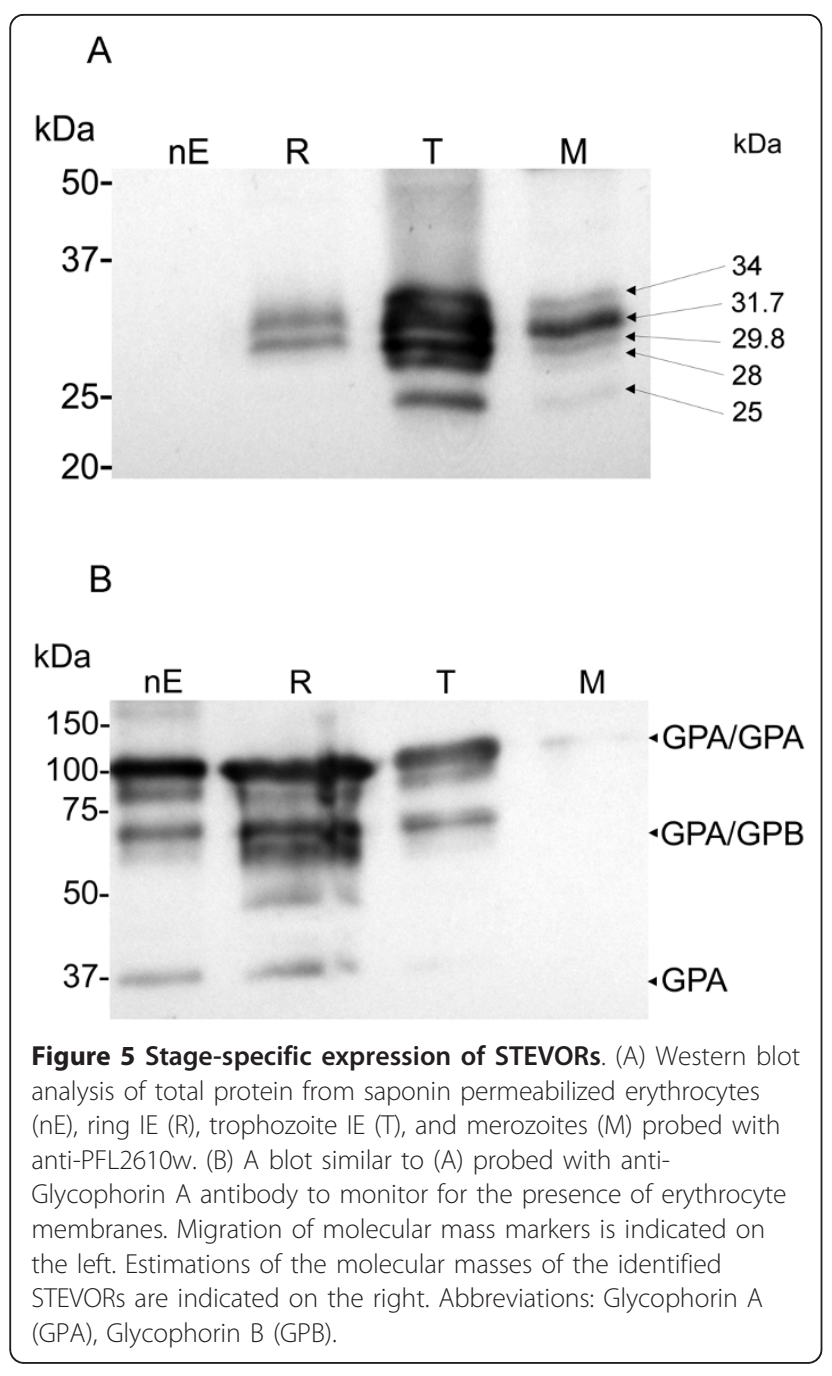

variant (PF10_0395 and PFF0850c) in flow cytometry assays [9]. In a previous study we also showed the association of STEVOR with schizont IE surface by the IEM technique [8].

In the current work, STEVORs were shown to be expressed on the surface of the merozoite stage. This is the first time that a variant antigen has been shown to be expressed on the surface of extracellular P. falciparum stage. Unexpectedly, the current study failed to detect STEVORs on the surface of schizont IE using the antiPFL2610w antibody which localized STEVORs to the surface of the merozoites. However, this antibody recognized STEVORs in internal structures after permeabilization of the schizont IE. These findings could simply indicate that the STEVOR variants recognized by antiPFL2610w antibody were not exposed on the surface of the schizont IE. Instead, other variants, if any, could be exposed. In a previous attempt to link stevor gene sequence diversity with surface expression STEVORs were detected on the surface of schizont IE using antibodies that were made against the conserved region only [9]. It was suggested by the latter study that the predicted transmembrane domain 1 is not inserted into the erythrocyte membrane. It was also suggested that the conserved region and the variable loop are exposed on the surface through anchoring of STEVOR as a single pass membrane protein. If that were the case, the antiPFL2610w antibody should have recognized the surface of the schizont IE as it was generated against both the conserved and the variable regions. One possible explanation for this conflict in reagent specificities relates to differences between the IgG fractions in the polyclonal antibodies that target the non-conserved epitopes on the conserved region of STEVORs. These IgG fractions should be also variant specific and their targets could be more exposed on the protein and lead to limited crossreactivity. It is also possible that STEVORs might not be exposed at all on the IE surface. This argument should remain valid until further evidence on surface expression is presented. Moreover, the noticeable expression of STEVORs in the schizont stage may only represent a step in the biogenesis process of the merozoite surface coat. Thus, the presence of STEVORs in the schizont stage does not mean that they should be exposed on the surface because they may not function at this stage. This resembles the appearance of MSP-1 at the schizont periphery at $34.5-36 \mathrm{~h}$ (p.i) in the biogenesis process of the merozoite surface coat [20] that will ultimately lead to exposure of MSP-1 on the merozoite surface, but not on the surface of the schizont IE.

Table 1 Quantification of STEVOR bands identified by the anti-PFL2610w in Western blot analysis

\begin{tabular}{lllllll}
\hline \multirow{2}{*}{ STEVOR variant $(\boldsymbol{k D a})$} & \multicolumn{2}{l}{ Ring infected erythrocytes } & \multicolumn{2}{l}{ Trophozoite infected erythrocytes } & \multicolumn{2}{l}{ Merozoites } \\
\cline { 2 - 7 } & Band intensity $^{1}$ & Relative intensity & Band intensity & Relative intensity & Band intensity & Relative intensity \\
\hline 34 & & 122 & 0.15 & 62 & 0.09 \\
31.7 & 0.56 & 159 & 0.25 & 181 & 0.65 \\
29.8 & 0.44 & 177 & 0.23 & 72 & 0.19 \\
28 & 93.60 & 155 & 0.18 & 20 & 0.04 \\
25 & & 162 & 0.19 & 17 & 0.03 \\
\hline
\end{tabular}

${ }^{1}$ The intensity value at the band peak.

${ }^{2}$ The percent intensity contribution of a band within a lane. 
Stage-specific expression of STEVOR variants was assessed by estimating the number and the level of expression of variants identified by the anti-PFL2610w antibody in the ring, trophozoite and merozoite stages. The study revealed an over-expression of a specific variant $(31.7 \mathrm{kDa})$ in the merozoites. This finding agrees with the postulation that the widespread expression of STEVOR in different stages is for serving different functions and indeed such a role should be played by stagespecific variants. RIFINs represented an example on stage-specific expression of distinct variants based on categorization of the encoding sequences and the corresponding protein localization [21]. In this report, RIFINs were grouped into two types, the A-type RIFIN that was found to be associated with the membrane of the IE or the apical region of the merozoites and the B-type RIFIN that was retained inside the parasite. Stage-specific expression of STEVOR variants based on differences in gene or upstream sequences has not been reported so far. In a transcription study stevor transcripts in the 3D7 trophozoites and gametocytes were similar except for a single stevor gene that was down regulated in gametocytes [22]. These findings suggest that some variants might only be expressed in certain stages to mediate a stage-specific function.

Moreover, an unexpected finding in the stage-specific expression of STEVORs was the detection of STEVORs (31.7 and $29.8 \mathrm{kDa}$ ) in the ring stage of the parasite. This is in contrast to the known first appearance of stevor transcripts at $22 \mathrm{~h}$ (p.i). It is likely that STEVORs located on the merozoite surface could have been carried into the erythrocytes during invasion and might have persisted for some time. Similar finding was also observed for the C-terminal 19-kDa fragment of the MSP-1 [20,23].

In conclusion, it was shown here that STEVORs are expressed on the surface of merozoites. This finding is in concordance with previous studies $[8,14]$ describing STEVORs expression in the merozoite stage of the $P$. falciparum parasite. Exposure of STEVORs on the merozoite surface may contribute to immune escape of merozoites for successful erythrocyte invasion.

\section{Acknowledgements}

We thank MJ Blackman, NIMR, London for anti-MSP-1 antibodies. This work received funding from the Sigrid Jusélius foundation and the Academy of Finland.

\section{Authors' contributions}

AK and SM conceived and designed the experiments. AK performed the experiments, analysed the data, and wrote the paper. SM participated in the revision of the paper. All authors read and approved the final manuscript.

\section{Competing interests}

The authors declare that they have no competing interests.
Received: 26 October 2010 Accepted: 14 March 2011

Published: 14 March 2011

\section{References}

1. Snow RW, Guerra CA, Noor AM, Myint HY, Hay SI: The global distribution of clinical episodes of Plasmodium falciparum malaria. Nature 2005, 434:214-217.

2. Marsh K, Kinyanjui S: Immune effector mechanisms in malaria. Parasite Immunol 2006, 28:51-60.

3. Langhorne J, Ndungu FM, Sponaas AM, Marsh K: Immunity to malaria: more questions than answers. Nat Immunol 2008, 9:725-732.

4. Schofield L, Mueller I: Clinical immunity to malaria. Curr Mol Med 2006, 6:205-221

5. Dzikowski R, Templeton TJ, Deitsch K: Variant antigen gene expression in malaria. Cell Microbiol 2006, 8:1371-1381.

6. Kaviratne M, Khan SM, Jarra W, Preiser PR: Small variant STEVOR antigen is uniquely located within Maurer's clefts in Plasmodium falciparuminfected red blood cells. Eukaryot Cell 2002, 1:926-935.

7. Lavazec C, Sanyal S, Templeton TJ: Hypervariability within the Rifin, Stevor and Pfmc-2TM superfamilies in Plasmodium falciparum. Nucleic Acids Res 2006, 34:6696-6707.

8. Khattab A, Bonow I, Schreiber N, Petter M, Schmetz C, Klinkert MQ: Plasmodium falciparum variant STEVOR antigens are expressed in merozoites and possibly associated with erythrocyte invasion. Malar J 2008, 7:137.

9. Niang $M$, Yan Y, Preiser PR: The Plasmodium falciparum STEVOR multigene family mediates antigenic variation of the infected erythrocyte. PLOS Pathog 2009, 5:e1000307.

10. McRobert L, Preiser P, Sharp S, Jarra W, Kaviratne M, Taylor MC, Renia L, Sutherland CJ: Distinct trafficking and localization of STEVOR proteins in three stages of the Plasmodium falciparum life cycle. Infect Immun 2004, 72:6597-6602.

11. Gardner MJ, Hall N, Fung E, White O, Berriman M, Hyman RW, Carlton JM, Pain A, Nelson KE, Bowman S, Paulsen IT, James K, Eisen JA, Rutherford K, Salzberg SL, Craig A, Kyes S, Chan MS, Nene V, Shallom SJ, Suh B, Peterson J, Angiuoli S, Pertea M, Allen J, Selengut J, Haft D, Mather MW, Vaidya AB, Martin DM, Fairlamb AH, Fraunholz MJ, Roos DS, Ralph SA, McFadden Gl, Cummings LM, Subramanian GM, Mungall C, Venter JC, Carucci DJ, Hoffman SL, Newbold C, Davis RW, Fraser CM, Barrell B: Genome sequence of the human malaria parasite Plasmodium falciparum. Nature 2002, 419:498-511.

12. Lavazec C, Sanyal S, Templeton TJ: Expression switching in the stevor and Pfmc-2TM superfamilies in Plasmodium falciparum. Mol Microbiol 2007 64:1621-1634.

13. Sam-Yellowe TY, Florens L, Johnson JR, Wang T, Drazba JA, Le Roch KG, Zhou Y, Batalov S, Carucci DJ, Winzeler EA, Yates JR III: A Plasmodium gene family encoding Maurer's cleft membrane proteins: structural properties and expression profiling. Genome Res 2004, 14:1052-1059.

14. Blythe JE, Yam XY, Kuss C, Bozdech Z, Holder AA, Marsh K, Langhorne J, Preiser PR: Plasmodium falciparum STEVOR proteins are highly expressed in patient isolates and located in the surface membranes of infected red blood cells and the apical tips of merozoites. Infect Immun 2008, 76:3329-3336.

15. Schreiber N, Khattab A, Petter M, Marks F, Adjei S, Kobbe R, May J, Klinkert MQ: Expression of Plasmodium falciparum 3D7 STEVOR proteins for evaluation of antibody responses following malaria infections in naive infants. Parasitology 2008, 135:155-167.

16. Scherf A, Lopez-Rubio JJ, Riviere L: Antigenic variation in Plasmodium falciparum. Annu Rev Microbiol 2008, 62:445-470.

17. Pasloske BL, Howard RJ: Malaria, the red cell, and the endothelium. Annu Rev Med 1994, 45:283-295.

18. Kyes SA, Rowe JA, Kriek N, Newbold Cl: Rifins: a second family of clonally variant proteins expressed on the surface of red cells infected with Plasmodium falciparum. Proc Natl Acad Sci USA 1999, 96:9333-9338.

19. Petter $M$, Haeggstrom M, Khattab A, Fernandez V, Klinkert MQ, Wahlgren M: Variant proteins of the Plasmodium falciparum RIFIN family show distinct subcellular localization and developmental expression patterns. $\mathrm{Mol}$ Biochem Parasitol 2007, 156:51-61.

20. Margos G, Bannister LH, Dluzewski AR, Hopkins J, Williams IT, Mitchell GH: Correlation of structural development and differential expression of 
invasion-related molecules in schizonts of Plasmodium falciparum.

Parasitology 2004, 129:273-287.

21. Petter M, Bonow I, Klinkert MQ: Diverse expression patterns of subgroups of the rif multigene family during Plasmodium falciparum gametocytogenesis. PLoS One 2008, 3:e3779.

22. Sharp S, Lavstsen T, Fivelman QL, Saeed M, McRobert L, Templeton TJ, Jensen AT, Baker DA, Theander TG, Sutherland CJ: Programmed transcription of the var gene family, but not of stevor, in Plasmodium falciparum gametocytes. Eukaryot Cell 2006, 5:1206-1214.

23. Blackman MJ, Heidrich HG, Donachie S, McBride JS, Holder AA: A single fragment of a malaria merozoite surface protein remains on the parasite during red cell invasion and is the target of invasion-inhibiting antibodies. J Exp Med 1990, 172:379-382.

doi:10.1186/1475-2875-10-58

Cite this article as: Khattab and Meri: Exposure of the Plasmodium

falciparum clonally variant STEVOR proteins on the merozoite surface.

Malaria Journal 2011 10:58.

\section{Submit your next manuscript to BioMed Central} and take full advantage of:

- Convenient online submission

- Thorough peer review

- No space constraints or color figure charges

- Immediate publication on acceptance

- Inclusion in PubMed, CAS, Scopus and Google Scholar

- Research which is freely available for redistribution

Submit your manuscript at www.biomedcentral.com/submit
(Ciomed Central 